\title{
The molecular orientation in Monomolecular Layer of Asymmetrically Substituted Copper (II) Phthalocyanines
}

\author{
Zhenxing Wang ${ }^{1}$, Haining Cui ${ }^{1{ }^{*}}$, Shiquan $\mathrm{Xi}^{2}$ \\ ${ }^{1}$ Department of Optical Information Science and Technology, College of Physics, College of \\ Zhaoqing (526061), Jilin University, Changchun(130012), P. R. China \\ ${ }^{2}$ Changchun Institute of Applied Chemistry, Chinese Academy of Sciences, Chang Chun 130022 \\ P. R. China \\ cuihaining2009@126.com
}

Keywords: Copper (II) Phthalocyanine film, Monomolecular Layer, structural characterization.

Abstract. Systematic research on monomolecular layer of asymmetrically substituted Copper (II) Phthalocyanine $(\mathrm{CuPc})$ and mixed $\mathrm{CuPc}$ with arachidic acid (AA) has been done. In this paper an attention is focused upon the molecular orientation in the film by means of the surface pressure-area $(\pi-\mathrm{A})$ isotherm method, polarized UV-visible and polarized infrared spectra spectroscopy.

\section{Introduction}

Phthalocyanine (Pc) derivatives, as highly conjugated and symmetric disk-shaped molecules, exhibit a wide variety of physical, chemical and electrical properties. The monolayer and nanometer assembly films of these macrocycle derivatives by means of Langmuir-Blogdett (LB) technique allow functional molecules to be arranged in a highly ordered structure and offer the possibility of application in many aspects[1-2]. In this paper, of particular interests are determination of the molecular orientation in the film.

\section{Experiments and Preparation of the film}

For improving the film forming properties the asymmetrically substituted copper phthalocyanine (CuPc) was synthesized and is shown in Table 1 . They were prepared according to the procedures reported by Snow et al [3]. Details of the preparation and characterization were described elsewhere[4]. KSV-5000 Langmuir trough (Finland) with a Wilhelmy balance and domestic LB trough were employed for the surface pressure-area $(\pi-A)$ isotherm measurements and the LB films fabrication. The monolayer was deposited when the substrate was moving upwards and downwards for Y type film. Polarized spectra of films were observed using a Shimadzu UV-VIS spectrophotometer (UV-3000). Infrared spectroscopy was employed BIO-RAD FTS-7 FT-IR spectrometer.

Table 1. Schematic structure of the asymmetrically substituted copper phthalocyanines ( $\mathrm{CuPc})$

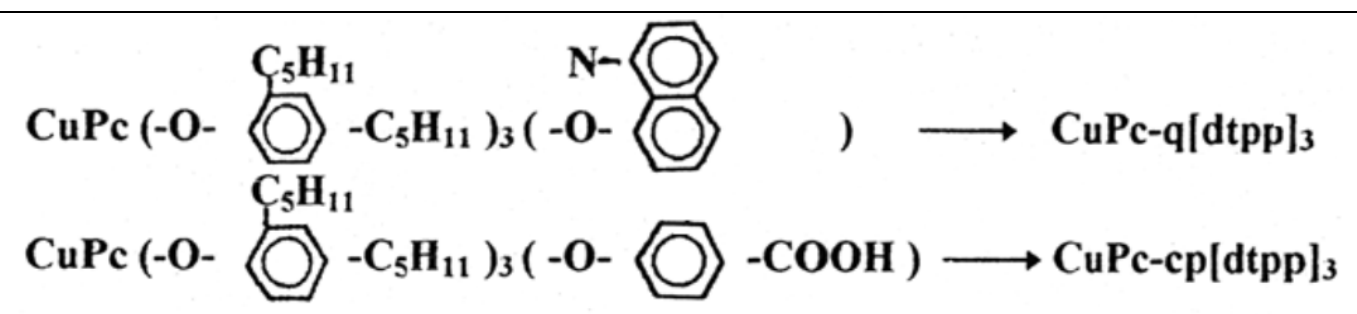




\section{Determination studies of the orientation of CuPc}

One of the striking characteristics of ordered organic molecular films is the orientation of the layer molecule. In order to deal with it, the phthalocyanine ring is considered as a flat circular plate on which

- * transition dipole is uniformly distributed according to Yoneyama[5]. The experimental data and calculation results are listed in Table 2 . In the equations and Table $2, \mathrm{~A}_{\|}$and $\mathrm{A}_{\perp}$ are the absorbance of the film for polarized light with electric vectors both parallel $(\|)$ and perpendicular $(\perp)$ to the dipping direction, respectively. The dichroic ratio $\mathrm{D}$ can be expressed as $\mathrm{A}_{\|} / \mathrm{A}_{\perp}$. The coordinates $(\mathrm{x}$, $\mathrm{y}, \mathrm{z})$ and incidence angles in the optical geometry are given as following: $\mathrm{y}$-axis is dipping direction of the film, z-axis is a normal line of the substrate of the film; the orientational angles $\theta$ is between $z$-axis and a normal line of $\mathrm{CuPc}$ macrocycles; $\phi$ is between $\mathrm{x}$-axis and $\mathrm{XY}$ plane projection of the normal line of $\mathrm{CuPc}$ macrocycles; the refraction angle $\beta=\sin ^{-1}\left(\mathrm{n}^{-1} \sin ^{-1} \beta^{\prime}\right)$ and refraction index in the film $\mathrm{n}=$ 1.39; $\mathrm{A}_{\|}$and $\mathrm{A}_{\perp}$ can be obtained from polarized UV-VIS spectra.

As we know, as the minimum plane area occupied by macrocycle of CuPc molecule would be 1.6 $\mathrm{nm}^{2}$ and the surface area of the edge is $0.4-0.8 \mathrm{~nm}^{2}$. According to Table 2, XY plane (substrate surface) projection area of the 'tilted' molecule on the substrate is about $1.60 \mathrm{~nm}^{2} \times \cos 38.1^{0} \cong 1.26 \mathrm{~nm}^{2}$ for $\mathrm{CuPc}-\mathrm{cp}[\mathrm{dtpp}]_{3}$; $\mathrm{XY}$ plane projection area of the 'tilted' molecule is about $1.60 \mathrm{~nm}^{2} \times \cos 60.2^{0} \cong 0.80$ $\mathrm{nm}^{2}$ for CuPc-q[dtpp $]_{3}$; XY plane projection area of the 'tilted' molecule is about $1.60 \mathrm{~nm}^{2} \times \cos 60.4^{0} \cong$ $0.80 \mathrm{~nm}^{2}$ for $\mathrm{CuPc}-\mathrm{q}[\mathrm{dtpp}]_{3}$ mixed with arachidic acid (AA). It suggests that mixing AA molecules into $\mathrm{CuPc}$ monolayer do change the angle of $\mathrm{CuPc}$ ring orientation in the monolayer.

Table.2 UV-vis experimental data and orientation calculations of CuPc ring in Y-type LB films of CuPc-cp $[\mathrm{dtpp}]_{3}$ and $\mathrm{CuPc}-\mathrm{q}[\mathrm{dtpp}]_{3}$ mixed with arachidic acid (AA)

\begin{tabular}{lcccc}
\hline & & CuPc-cp[dtpp $]_{3}$ & CuPc-q[dtpp $]_{3}$ & $\begin{array}{c}\text { CuPc-q[dtpp] }]_{3} \text { mixed } \\
\text { with AA }(1: 1)\end{array}$ \\
\hline$\beta^{\prime}=0^{0}$ & $\mathrm{~A}_{\|}$ & 0.314 & 0.222 & 0.237 \\
& $\mathrm{~A}_{\perp}$ & 0.336 & 0.223 & 0.237 \\
& $\mathrm{D}_{\beta}$ & 0.935 & 0.996 & 1.000 \\
\hline & $\mathrm{A}_{\|}$ & 0.353 & 0.226 & 0.250 \\
$\beta^{\prime}=30^{0}$ & $\mathrm{~A}_{\perp}$ & 0.351 & 0.233 & 0.257 \\
& $\mathrm{D}_{\beta}$ & 1.006 & 0.970 & 0.973 \\
\hline$<\cos ^{2} \theta>$ & 0.620 & 0.246 & 0.244 \\
$<\theta>$ & $38.1^{0}$ & $60.2^{0}$ & $60.4^{0}$ \\
\hline
\end{tabular}

According to the area per molecule found in our study, the monomolecular area for CuPc in film are less than that of real $\mathrm{CuPc}$ ring area. The measured data and plus calculation of $\mathrm{CuPc}$ area in the film is 0.80 and $1.26 \mathrm{~nm}^{2}$ is incompatible with an arrangement of the macrocycle parallel to the water surface (or substrate surface) in which area value should be near $1.6 \mathrm{~nm}^{2}$. In the same reason the rings are not perpendicular to the surface either. This indicates a tilted of the planes of $\mathrm{CuPc}$ macrocycle with respect to one another.

The molecular orientation of various Pc films could be studied by the information of area per molecule from $\pi$-A isotherm. Fig. 1 shows $\pi$-A isotherm of CuPc-q[dtpp $]_{3}$ and mixed CuPc-q[dtpp $]_{3}$ with fatty acid molecule. As can be seen in Fig.1, a stable mixed monolayer composed of CuPc-q[dtpp $]_{3}$ and AA was formed at various kinds of molar fraction. It can also be seen that the experimental values of average molecular area are less than the calculated values, which maybe explained as that $\mathrm{CuPc}-\mathrm{q}[\mathrm{dtpp}]_{3}$ is not dispersed as a single molecule within a AA matrix, but rather as 
aggregates in the mixed film. Above the molar ratio of $1: 1\left\{\mathrm{CuPc}-\mathrm{q}[\mathrm{dtpp}]_{3}: \mathrm{AA}\right\}$, the collapse pressure decreases with increasing molar fraction of $\mathrm{CuPc}$, while below this ratio, the collapse pressure remains almost constant, which is larger than that of pure AA monolayer. These reveal that film-forming ability of CuPc improves. The above mixed LB film results of polarized spectra (table 2) support these conclusions.

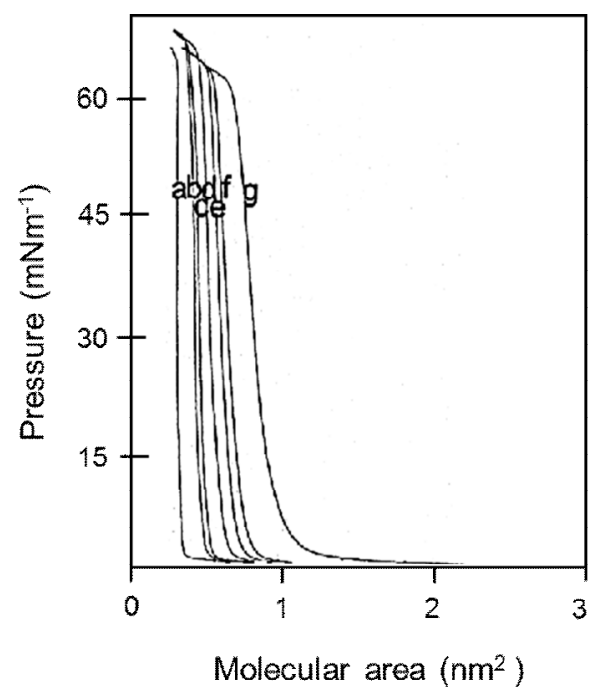

Fig. $1 \mathrm{Pi}-\mathrm{A}$ isotherms of $\mathrm{CuPc}$ mixed with AA in different mole fraction. (Temperature: $20+0.5$ 0C) a--Pure $\quad$ AA; $\quad$ b--AA:CuPc(3:1); c--AA:CuPc(2:1); d--AA:CuPc(1:1); e--AA:CuPc(1:2); f--AA:CuPc(1:3); g--Pure CuPc.

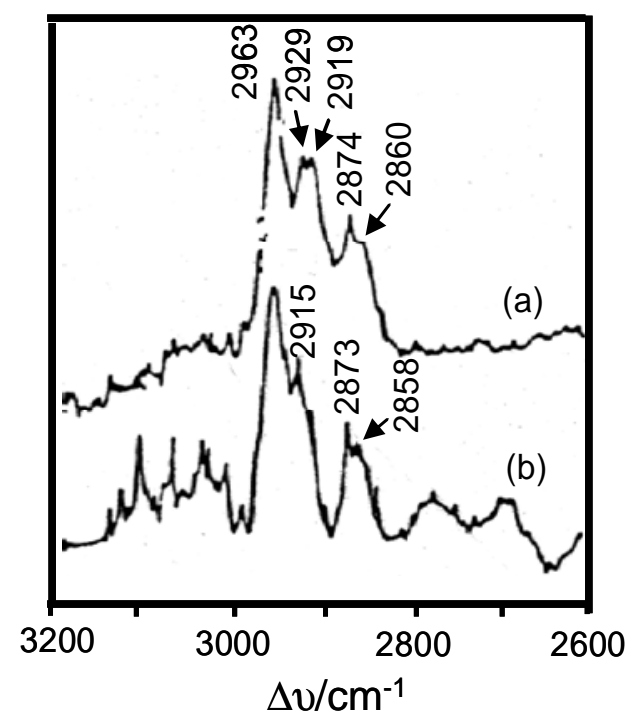

Fig.2 (a) (b) Polarized infrared transmission spectra of CuPc-q[dtpp]3. The 30-monolayer film measured by using polarized light with the electric vectors parallel and perpendicular to the direction of dipping of the LB films.

Fig. 2 exhibits polarized infrared transmission spectra of the 30 -monolayer CuPc-q[dtpp $]_{3}$ film. The polarized light with the electric vectors is parallel and perpendicular to the direction of dipping of the LB films respectively. By comparing the results of sublimated film, in the case of ordered monomolecular film there is a splitting of $\mathrm{CH}_{2}$ antisymmetric stretching band, in which it becomes $2929 \mathrm{~cm}-1$ and $2919 \mathrm{~cm}-1$ (Fig.2a). For two spectra of sublimated film there is no dichroism of the IR 
bands observed. This suggests that two hydrocarbon chains of the benzene could be realized and poses an orientation characteristic in ordered LB film.

\section{Conclusion}

The present study provides insight into the determination of molecular orientation and structural characterization of ordered monomolecular layer by polarized spectra. For films of CuPc-q[dtpp]3 and mixed CuPc-q[dtpp] 3 with AA, the macrocycle plane of the $\mathrm{CuPc}$ is tilted with an angle about 400 in tendency of parallel to the substrate plane; for films of CuPc-cp[dtpp]3, the macrocycle plane of the $\mathrm{CuPc}$ is tilted with an angle about 600 in tendency of parallel to the normal line of substrate plane. Asymmetrically substituted CuPc have better forming properties of LB film. Some mixing fatty acid can improve the film-firming ability, while the orientation angle of molecules is influenced.

\section{Acknowledgements}

Authors would like to thank Prof. W. Q. Chen for his help and Dr. Y. Wang for his partial experiment data. We acknowledge partial financial supports from Jointly Funded Project (No. 61179055) of Chinese Civil Aviation Authority and National Natural Science Foundation of China, Talent Grant (2013-ZQXY-05) of Educational Commission of Guangdong Province, China

\section{References}

[1] K. P. Gentry, G. Thomas: (2009) Phys. Rev. B , 80:174118

[2] A. Capobianchi, A. M. Paoletti, G. Rossi, G. Zanotti, G. Pennesi: ( 2009), Sensors and Actuators B: Chemical 142 (1): 159.

[3] A. W. Snow and N. L. Jarvis: J. Am. Chem. Soc., 1984, 106, 4706

[4] Y. Zang; W. Q. Chen and Q. Shen: Chem. J.of Chinese Universities, 1993, 11, 1483 and 1995, 11, 547

[5] Yoneyama,M.; Sugi, M. J. Appl. Phys., 1986, 25, 961 